\title{
Clinical Significance of Atopic Dermatitis with Hypoalbuminemia in
} Korean Children

\author{
Chanho Lee, ${ }^{1}$ Songhan Lee, ${ }^{1}$ Sung Won Kim, ${ }^{1}$ and Myongsoon Sung, ${ }^{2,}$ \\ ${ }^{1}$ Department of Pediatrics, Busan St. Mary's Hospital, Busan, Korea \\ ${ }^{2}$ Department of Pediatrics, CHA Gumi Medical Center, CHA University School of Medicine, Gumi, Korea \\ "Corresponding author: Myongsoon Sung, Ph.D, Department of Pediatrics, CHA Gumi Medical Center, CHA University School of Medicine, 855 Hyeonggokdong Gumi \\ Gyeongsangbukdo 730-728. Tel: +82-544509700, E-mail: myong47@hanmail.net
}

Received 2016 June 28; Revised 2016 November 28; Accepted 2016 December 20.

\begin{abstract}
Background: Hypoalbuminemia can be a life-threatening complication of severe atopic dermatitis (AD).

Objectives: The aim of this study was to evaluate correlations between clinical features and laboratory tests of AD children with scoring atopic dermatitis (SCORAD) scores $\geq 40$, according to the presence of hypoalbuminemia.

Methods: Children with AD between 3 and 24 months of age with SCORAD score $\geq 40(n=82)$, admitted to our unit from June 2007 to March 2016, were categorized to two groups of hypoalbuminemic $(n=27)$ and non-hypoalbuminemic $(n=55)$. A blood albumin level of $\leq 3.5 \mathrm{~g} / \mathrm{d}$ on the first day of admission was considered as hypoalbuminemia. The results of clinical and laboratory tests of the two groups were evaluated and compared.

Results: Significant differences were observed in different genders, age at AD onset, and duration of AD between the groups. Compared with non-hypoalbuminemia group, significantly more patients in hypoalbuminemia group had positive test results for methicillin-resistant Staphylococcus aureus (MRSA) as well as allergen sensitization $(\mathrm{P}<0.05)$. After adjusting for age and gender, male gender (odds ratio (OR) 5.962; 95\% confidence interval (CI) $2.136-16.644, \mathrm{P}=0.001$ ), positive MRSA (OR, 10.625; $95 \% \mathrm{CI}$, 2.823 $39.982, \mathrm{P}<0.001)$ and allergen $(\mathrm{OR}, 4.622 ; 95 \% \mathrm{CI}, 1.573-13.578, \mathrm{P}=0.005)$ test results were strongly related to the presence of hypoalbuminemia.

Conclusions: Hypoalbuminemia in AD children with SCORAD score $\geq 40$ is associated with increased complications.
\end{abstract}

Keywords: Atopic dermatitis, Hypoalbuminemia, Children

\section{Background}

Atopic dermatitis (AD), perpetuated by geneenvironmental reciprocal action, is the most common type of dermatosis in infants and children, and has the characteristics of genetic barrier defects and allergic inflammation $(1,2)$. The number of patients with $\mathrm{AD}$ is continuing to increase in many developed and developing countries $(3,4)$. According to the International Study of asthma and allergies in childhood (ISAAC), the prevalence of $\mathrm{AD}$ is $10 \%$ to $20 \%$ in children and $1 \%$ to $3 \%$ in adults worldwide (2). The lifetime prevalence of pruritus eczema in children aged 6 to 7 years has increased from $17.1 \%$ to $27.0 \%$, while within 12 months this prevalence has increased from $13.4 \%$ to $20.6 \%(5,6)$. Because of the increasing prevalence of $\mathrm{AD}$, complications from recurrent bacterial and viral skin infections are also on the rise (7). Atopic Dermatitis complications are usually not lifethreatening conditions, yet, a few associated conditions, such as hypoalbuminemia, could be dangerous to patients $(8,9)$.

Hypoalbuminemia is caused by epidermal permeability barrier defects in patients with AD and may occur when taking herbal medicine or restricting milk formula for AD treatment in children (10). Hypoalbuminemia is usually accompanied by hypoproteinemia and thrombocytosis. As a result, hypoalbuminemia can be a life-threatening condition owing to hypovolemic shock combined with hypoproteinemia and vascular infarction caused by thrombocytosis (11-13).

However, because hypoalbuminemia has a low prevalence, there have been only a few studies of hypoalbuminemia in children with AD.

\section{Objectives}

The aim of this study was to evaluate correlations between clinical features and laboratory test results of $\mathrm{AD}$ children with scoring atopic dermatitis (SCORAD) score of $\geq 40$, according to the presence of hypoalbuminemia.

\section{Methods}

\subsection{Participants}

The study population consisted of patients between 3 and 24 months of age, referred to the pediatric allergy and 
respiratory center at Mary's Hospital, Busan, South Korea, because of an itching sensation, dry skin, weeping, and edema. These patients were diagnosed with severe AD by allergy specialists between June 2007 and March 2016. The study was approved by the institutional review board at St. Mary's Hospital in Busan, South Korea.

Diagnosis of AD was made in accordance with the diagnostic criteria proposed by Hanifin and Rajka (14) and the SCORAD score was calculated by allergy specialists. Moderate to severe $\mathrm{AD}$ was defined as SCORAD score of $\geq 40$ (15). A blood albumin level of $\leq 3.5 \mathrm{~g} / \mathrm{dL}$ (16) on the first day of admission was considered as hypoalbuminemia. Patients with chronic or kidney diseases (bronchopulmonary dysplasia, congestive heart disease, nephrotic syndrome, etc.) were excluded from the study. Children with moderate to severe $\mathrm{AD}(\mathrm{n}=82)$ were categorized to two groups, hypoalbuminemia $(\mathrm{n}=27)$ and non-hypoalbuminemia $(\mathrm{n}=55)$.

\subsection{Laboratory Tests}

The levels of Eosinophil Cationic protein (ECP), total immunoglobulin E (IgE), and specific $\operatorname{IgE}(\mathrm{s} \operatorname{IgE})$, as well as peripheral blood eosinophil count were assayed on the first day of admission. Levels of ECP, total IgE, and SIgE for allergens were measured using Immuno CAP 250 (Thermo Fisher, Uppsala, Sweden). A sIgE level of $>0.7 \mathrm{kUA} / \mathrm{L}$ was used to define sensiti-vity to house dust mites (Dermatophagoides pteronyssinus and Dermatophagoides farinae) and foods (eggs, milk, soybeans, and peanuts). Peripheral blood eosinophil counts were measured using an automated hematology analyzer (Coulter Counter STKS; Beckman Coulter, Fullerton, CA, USA), using blood samples collected in Ethylenediaminetetraacetic Acid (EDTA) tubes.

\subsection{Skin Culture}

Before AD treatment, a skin swab test was taken from inflamed, pus-filled, or oozing areas. One swab was collected per patient and transported to the laboratory. Swabs were inoculated directly onto blood agar plates and incubated at $35^{\circ} \mathrm{C}$ for 24 hours before being examined. If there were specific yellow colonies, a test of catalase and coagulase using VITEC 2 (Biomerius Inc., Durham, NC, USA) was conducted to classify Staphylococcus aureus. After using VITEC 2, according to the national committee for clinical laboratory standards, the minimal inhibitory concentration (MIC) was also determined. If the MIC to oxacillin was $\geq 4 \mu \mathrm{g} / \mathrm{mL}$, S. aureus colonies were classified as MethicillinResistant S. aureus (MRSA).

\subsection{Statistical Analysis}

All statistical analyses were performed using IBM SPSS version 21.0 (IBM Co., Armonk, NY, USA). The experiment-tal results are presented as arithmetic mean \pm standard deviation. One-way analysis of variance was used for continuous variables to assess intergroup differ-ences. Multivariate logistic regression analysis was used to identify risk factors for hypoalbuminemia, and Odds Ratios (OR) and 95\% Confidence Interval (CI) were calculated. Values of $\mathrm{P}<0.05$ were considered statistically significant.

\section{Results}

The clinical characteristics of both groups are shown in Table 1. Significant differences were observed in gender, average age, age at $\mathrm{AD}$ onset, and duration of $\mathrm{AD}$ between the groups. Hypoalbuminemia group was extremely male dominant, whereas non-hypoalbuminemia group had approximately equal proportion of males and females. No significant differences were observed in delivery method, average body weight, average body weight percentile, parental allergic disease history, or SCORAD score $(\mathrm{P}>0.05)$.

The laboratory test results of both groups are shown in Table 2. Serum white blood cell and platelet counts were significantly higher in hypoalbuminemia group. Serum C-reactive protein, total protein, and albumin levels were lower in hypoalbuminemia group, yet, the difference was significant only for albumin. Serum total IgE and ECP levels, and total eosinophil counts were significantly higher in the hypoalbuminemia group $(\mathrm{P}<0.001)$. Concerning positive skin culture results, there was no statistically significant difference between the groups $(\mathrm{P}=0.526)$. However, the hypoalbuminemia group had a significantly higher proportion of positive MRSA results than nonhypoalbuminemia group $(\mathrm{P}=0.008)$.

The allergy laboratory test results of both groups are shown in Table 3. The hypoalbuminemia group had a significantly higher proportion of positive test results for allergens. The hypoalbuminemia group also had a significantly higher proportion of children with multiple allergen sensitizations. The hypoalbuminemia group had a significantly higher proportion of children with milk, eggs, peanuts, soybeans, or house dust mite allergen sensitizations. Furthermore, the levels of specific IgEs to milk, eggs, peanuts, soybeans, and house dust mite were significantly higher in the hypoalbuminemia group.

The relationship between the development of hypoalbuminemia and associated factors is shown in Table 4. After adjusting for age and gender, male gender (odds ratio (OR) 5.962; 95\% confidence interval (CI) 2.136 to 16.644, P $=0.001$ ) and, positive MRSA (OR, 10.625; 95\% CI, 2.823 to 39.982, $\mathrm{P}<0.001)$ and allergen (OR, 4.622; 95\% CI, 1.573 to 13.578, $\mathrm{P}=0.005$ ) test results were strongly related to the presence of hypoalbuminemia. 
Table 1. Clinical Characteristics of the Study Groups ${ }^{\mathrm{a}}$

\begin{tabular}{|c|c|c|c|}
\hline Parameters & Hypoalbuminemia, $(\mathbf{n}=27)$ & Non- hypoalbuminemia, $(n=55)$ & P Value \\
\hline Gender, No. (\%) & & & 0.002 \\
\hline Male & $24(88.9)$ & $30(54.5)$ & \\
\hline Female & $3(11.1)$ & $25(45.5)$ & \\
\hline Age, mo & $7.22 \pm 2.90$ & $10.78 \pm 6.15$ & $<0.001$ \\
\hline Vaginal delivery, No. (\%) & $16(59.3)$ & $32(58.2)$ & 0.926 \\
\hline Weight, kg & $8.15 \pm 1.57$ & $9.35 \pm 1.65$ & 0.884 \\
\hline Weight percentile & $40.37 \pm 31.84$ & $56.31 \pm 27.51$ & 0.467 \\
\hline Parental allergic disease, №. (\%) & $22(81.5)$ & $37(67.3)$ & 0.178 \\
\hline Allergic rhinitis & $14(51.9)$ & $29(52.7)$ & 0.941 \\
\hline Atopic dermatitis & $3(11.1)$ & $8(14.5)$ & 0.668 \\
\hline Age at AD onset, mo & $4.44 \pm 2.41$ & $8.38 \pm 5.34$ & $<0.001$ \\
\hline Duration of AD, No. (\%) & & & 0.018 \\
\hline$<1$ years & $27(100)$ & $45(81.8)$ & \\
\hline$\geq 1$ years & $0(0.0)$ & $10(18.2)$ & \\
\hline SCORAD score & $53.73 \pm 13.87$ & $53.36 \pm 10.68$ & 0.386 \\
\hline
\end{tabular}

bbreviations: AD, atopic dermatitis; SCORAD, Scoring Atopic Dermatitis.

${ }^{a}$ Values are presented as mean \pm standard deviation.

Table 2. Comparison of Laboratory Test Results Between Hypoalbuminemia and Non-Hypoalbuminemia Groups ${ }^{\mathrm{a}}$

\begin{tabular}{|c|c|c|c|}
\hline Parameters & Hypoalbuminemia, $(n=27)$ & Non- hypoalbuminemia, $(n=55)$ & P Value \\
\hline White blood cell count, /mL & $23,617.81 \pm 6,424.56$ & $11,131.27 \pm 4,103.35$ & 0.033 \\
\hline Platelet, $10^{3} / \mu \mathbf{L}$ & $677.15 \pm 206.24$ & $395.38 \pm 119.49$ & 0.037 \\
\hline C-reactive protein, $\mathrm{mg} / \mathrm{dL}$ & $2.62 \pm 6.02$ & $2.80 \pm 5.72$ & 0.971 \\
\hline Total protein, $\mathbf{g} / \mathbf{d L}$ & $4.55 \pm 0.59$ & $6.36 \pm 0.45$ & 0.246 \\
\hline Albumin, $\mathbf{g} / \mathbf{d L}$ & $2.88 \pm 0.45$ & $4.32 \pm 0.23$ & $<0.001$ \\
\hline Total IgE, IU/mL & $1,066.07 \pm 921.04$ & $220.71 \pm 496.59$ & $<0.001$ \\
\hline $\mathbf{E C P}, \mu \mathrm{g} / \mathbf{L}$ & $121.39 \pm 83.83$ & $23.08 \pm 26.86$ & $<0.001$ \\
\hline Total eosinophil count, $\mid \mu \mathbf{L}$ & $5,648.52 \pm 3,616.18$ & $784.19 \pm 777.87$ & $<0.001$ \\
\hline Positive skin culture, No. (\%) & $26(96.2)$ & $51(92.7)$ & 0.526 \\
\hline MRSA & $11(40.7)$ & $8(14.5)$ & 0.008 \\
\hline MSSA & $13(48.1)$ & $16(29.1)$ & 0.090 \\
\hline
\end{tabular}

Abbreviations: ECP, Eosinophil Cationic Protein; Ige, Immunoglobulin E; MRSA, Methicillin-Resistant Staphylococcus Aureus; MSSA, Methicillin-Sensitive Staphylococcus Aureus.

${ }^{\mathrm{a}}$ Values are presented as mean \pm standard deviation.

\section{Discussion}

According to the presence of hypoalbuminemia, the data presented here shows a clear difference in clinical and laboratory test results in children with moderate to severe $\mathrm{AD}$. Almost $90 \%$ of the children with hypoalbuminemia were male, and younger at the onset of $\mathrm{AD}$, and had a shorter $\mathrm{AD}$ duration compared with the children without hypoalbuminemia. One would expect longer duration of $\mathrm{AD}$ in hypoalbuminemia group because of complications of allergen sensitization and increased MRAS.
However, our findings that indicate hypoalbuminemia occurred mainly in male infants with $\mathrm{AD}$, who were diagnosed recently, correlates with previous studies (9-13).

Almost all of the children with hypoalbuminemia in previous studies had an extremely low body weight. However, children with hypoalbuminemia in this study had almost average weight percentile, and 5 of 27 children weighed within the third percentile (9-13). Therefore, we concluded that children with normal body weight could also have hypoalbuminemia as an AD complication.

Hypoalbuminemia can occur with hypoproteinemia 
Table 3. Comparison of Allergic Laboratory Test Results Between Hypoalbuminemia and Non-Hypoalbuminemia Groups ${ }^{\mathrm{a}}$

\begin{tabular}{|c|c|c|c|}
\hline Parameters & Hypoalbuminemia, $(\mathbf{n}=27)$ & Non- Hypoalbuminemia, $(n=55)$ & P Value \\
\hline Numbers of positive to allergen, No. (\%) & $26(96.3)$ & $28(50.9)$ & $<0.001$ \\
\hline Mono & $1(3.7)$ & $8(14.5)$ & \\
\hline Poly & $25(92.6)$ & $20(36.4)$ & \\
\hline \multicolumn{4}{|l|}{ Positive to allergen, No. (\%) } \\
\hline Eggs & $23(85.2)$ & $26(47.3)$ & 0.001 \\
\hline Milk & $22(81.5)$ & $14(25.5)$ & $<0.001$ \\
\hline Peanuts & $19(70.4)$ & $10(18.2)$ & $<0.001$ \\
\hline Soybean & $21(77.8)$ & $12(21.8)$ & $<0.001$ \\
\hline HDM & $16(59.3)$ & $4(7.3)$ & $<0.001$ \\
\hline \multicolumn{4}{|l|}{ Specific IgE (kUA/L) } \\
\hline Specific IgE to eggs & $52.97 \pm 39.65$ & $11.54 \pm 25.52$ & $<0.001$ \\
\hline Specific IgE to milk & $22.91 \pm 32.46$ & $3.72 \pm 12.65$ & $<0.001$ \\
\hline Specific IgE to peanuts & $30.45 \pm 35.15$ & $3.52 \pm 11.10$ & $<0.001$ \\
\hline Specific IgE to soybean & $28.07 \pm 39.91$ & $3.12 \pm 5.15$ & $<0.001$ \\
\hline Specific IgE to HDM & $12.77 \pm 28.39$ & $3.76 \pm 18.88$ & $<0.001$ \\
\hline
\end{tabular}

Abbreviations: HDM, House Dust Mite; IgE, Immunoglobulin E.

${ }^{\mathrm{a}}$ Values are presented as mean \pm standard deviation.

Table 4. The Relationship Between the Development of Hypoalbuminemia and Associated Factors

\begin{tabular}{|c|c|c|c|c|}
\hline Variable & OR $(95 \% \mathrm{CI})$ & PValue & $\operatorname{aOR}(95 \% \mathrm{CI})^{\mathrm{a}}$ & P Value \\
\hline Male gender & $6.667(2.446-18.169)$ & $<0.001$ & $5.962(2.136-16.644)$ & 0.001 \\
\hline Age. mo & $1.142(1.037-1.259)$ & 0.007 & $1.129(1.016-1.254)$ & 0.024 \\
\hline Total IgE, IU/mL & $0.998(0.998-0.999)$ & $<0.001$ & $0.999(0.998-0.999)$ & 0.002 \\
\hline $\mathbf{E C P}, \mu \mathbf{g} / \mathbf{L}$ & $0.976(0.967-0.985)$ & $<0.001$ & $0.976(0.964-0.987)$ & $<0.001$ \\
\hline Positive MRSA & $4.727(1.805-12.382)$ & 0.002 & $10.625(2.823-39.982)$ & $<0.001$ \\
\hline Positive allergen & $14.718(3.284-65.964)$ & $<0.001$ & $4.622(1.573-13.578)$ & 0.005 \\
\hline
\end{tabular}

$(9,11,13)$. Thrombocytosis was recently described as a complication of severe childhood AD with hypoproteinemia (17-19). Hypovolemia associated with hypoproteinemia may also play a role in increased platelet counts and increase the risk of thrombosis. In other studies, an infant with severe AD and hypoalbuminemia had leukocytosis, and $>60 \%$ of the patients with hypoproteinemia also had thrombocytosis and leukocytosis $(11,17)$. The studies also showed that almost $90 \%$ of children with hypoalbuminemia had thrombocytosis and leukocytosis.

In a previous study, $\mathrm{AD}$ children with hypoalbuminemia or hypoproteinemia had high serum total IgE and eosinophil counts as well as specific IgE antibodies to food (11). In the present study, children with hypoalbuminemia had higher serum total IgE and ECP levels, and eosinophil counts than those without hypoalbuminemia. There must be more possible mechanisms by which children with moderate to severe $\mathrm{AD}$ with hypoalbuminemia develop elevated IgE or ECP or eosinophil count. However, due to the retrospective nature of the study we could not clarify the acting mechanisms. Therefore, more studies are needed to evaluate these mechanisms.

The children with hypoalbuminemia had very strong sensitivities to milk and eggs in this study. The presence 
of food allergy or diet restriction is an important factor in evaluating the relationship among hypoalbuminemia, food allergy, and diet in children with severe AD. However, our study design was not able to provide information about presence of food allergy or diet restriction.

Concerning skin culture results, children with AD with hypoalbuminemia or without hypoalbuminemia had high numbers of cutaneous infections in this study. Children with $\mathrm{AD}$ have high rates of $S$. aureus infection compared with those without $\operatorname{AD}(20,21)$. The two groups in this study did not have significant differences in positive skin results. However, MRSA infection that was more common in the children with hypoalbuminemia was strongly associated with the presence of hypoalbuminemia. Therefore, we assumed that MRSA infection influences skin barrier, and, as a result, hypoalbuminemia appears. More studies are needed to evaluate the relationship between $\mathrm{AD}$ and hypoalbuminemia, and MRSA infection.

The current study had a few limitations. Firstly, only 27 subjects with SCORAD score $\geq 40$ with hypoalbuminemia, were between 3 and 24 months of age, which is not a large number. Hypoalbuminemia is not a common complication of $\mathrm{AD}$ and we included only those children, who visited the allergy center over 9 years. Therefore, we could not obtain more subjects although 27 subjects are not too small to reach objectivity. Secondly, owing to its retrospective nature, we could not obtain enough information about the presence of food allergy or diet restriction.

However, this study has identified characteristics of children with SCORAD score of $\geq 40$ with hypoalbuminemia, including clinical features and laboratory test results. In other words, male gender, positive MRSA, and allergen are factors influencing hypoalbuminemia in Korean AD children. These findings suggest that $\mathrm{AD}$ in children with SCORAD score $\geq 40$ and hypoalbuminemia is associated with increased complications.

\section{References}

1. Lee SI, Kim J, Han Y, Ahn K. A proposal: Atopic Dermatitis Organizer (ADO) guideline for children. Asia Pac Allergy. 2011;1(2):53-63. doi: 10.5415/apallergy.2011.1.2.53. [PubMed: 22053298].

2. Schneider L, Tilles S, Lio P, Boguniewicz M, Beck L, LeBovidge J, et al. Atopic dermatitis: a practice parameter update 2012. J Allergy Clin Immunol. 2013;131(2):295-9 e1-27. doi: 10.1016/j.jaci.2012.12.672. [PubMed: 23374261].

3. Eder W, Ege MJ, von Mutius E. The asthma epidemic. $N$ Engl J Med. 2006;355(21):2226-35. doi: 10.1056/NEJMra054308. [PubMed: 17124020].

4. European Academy of Allergy and Clinical Immunology. Global Atlas of Allergy 2014 Available from: www.eaaci.org.
5. Oh JW, Pyun BY, Choung JT, Ahn KM, Kim CH, Song SW, et al. Epidemiological change of atopic dermatitis and food allergy in school-aged children in Korea between 1995 and 2000. J Korean Med Sci. 2004;19(5):716-23. doi: 10.3346/jkms.2004.19.5.716. [PubMed: 15483350].

6. Park YM, Lee SY, Kim WK, Han MY, Kim J, Chae Y, et al. Risk factors of atopic dermatitis in Korean schoolchildren: 2010 international study of asthma and allergies in childhood. Asian Pac J Allergy Immunol. 2016;34(1):65-72. doi: 10.12932/AP0621.34.1.2016. [PubMed: 26994628].

7. Boguniewicz M, Leung DY. Recent insights into atopic dermatitis and implications for management of infectious complications. J Allergy Clin Immunol. 2010;125(1):4-13. doi:10.1016/j.jaci.2009.11.027.[PubMed: 20109729] quiz 14-5.

8. Katoh N, Hosoi H, Sugimoto T, Kishimoto S. Features and prognoses of infantile patients with atopic dermatitis hospitalized for severe complications. J Dermatol. 2006;33(12):827-32. doi: 10.1111/j.13468138.2006.00190.x. [PubMed: 17169084].

9. Yasutomi M, Okazaki S, Kawakita A, Hayashi H, Murai H, Mayumi M, et al. [Case of atopic dermatitis in infant treated with Chinese herbal medicines and nsaids ointment, which induced weight loss, electrolyte disturbance and hypoproteinemia]. Arerugi. 2013;62(7):82732. [PubMed: 24129659].

10. Lee HJ, Lee SH. Epidermal permeability barrier defects and barrier repair therapy in atopic dermatitis. Allergy Asthma Immunol Res. 2014;6(4):276-87. doi: 10.4168/aair.2014.6.4.276. [PubMed: 24991450].

11. Goodyear HM, Harper JI. Atopic eczema, hyponatraemia, and hypoalbuminaemia. Arch Dis Child. 1990;65(2):231-2. [PubMed: 2317073].

12. Capulong MC, Kimura K, Sakaguchi N, Kawahara H, Matsubara K, Iikura Y. Hypoalbuminemia, oliguria and peripheral cyanosis in an infant with severe atopic dermatitis. Pediatr Allergy Immunol. 1996;7(2):100-2. doi: 10.111//j.1399-3038.1996.tb00114.x. [PubMed: 8902861].

13. Keller MD, Shuker M, Heimall J, Cianferoni A. Severe malnutrition resulting from use of rice milk in food elimination diets for atopic dermatitis. Isr Med Assoc J. 2012;14(1):40-2. [PubMed: 22624441].

14. Hanifin JM, Rajka G. Diagnostic features of atopic dermatitis. Acta Derm Venereol Suppl (Stockh). 1980;92.

15. Oranje AP, Glazenburg EJ, Wolkerstorfer A, de Waard-van der Spek FB. Practical issues on interpretation of scoring atopic dermatitis: the SCORAD index, objective SCORAD and the three-item severity score. Br J Dermatol. 2007;157(4):645-8. doi: 10.1111/j.1365-2133.2007.08112.x. [PubMed: 17714568].

16. Child CG, Turcotte JG. Surgery and portal hypertension. 3rd ed. ; . p. 90.

17. Nomura I, Katsunuma T, Tomikawa M, Shibata A, Kawahara H, Ohya Y, et al. Hypoproteinemia in severe childhood atopic dermatitis: a serious complication. Pediatr Allergy Immunol. 2002;13(4):287-94. doi: 10.1034/j.1399-3038.2002.01041.x. [PubMed: 12390445].

18. Abrahamov A, Schifmann R, Goldstein R, Tal Y, Freier S. Growth failure due to protein loss in dermatitis. Eur J Pediatr. 1986;145(3):223-6. doi: 10.1007/BF00446072. [PubMed: 3769980].

19. Novembre E, Leo G, Cianferoni A, Bernardini R, Pucci N, Vierucci A. Severe hypoproteinemia in infant with AD. Allergy. 2003;58(1):88-9. doi: 10.1034/j.1398-9995.2003.23710_6.x. [PubMed: 12580819].

20. Ong PY. Recurrent MRSA skin infections in atopic dermatitis. J Allergy Clin Immunol Pract. 2014;2(4):396-9. doi: 10.1016/j.jaip.2014.04.007. [PubMed: 25017526].

21. Jagadeesan S, Kurien G, Divakaran MV, Sadanandan SM, Sobhanakumari K, Sarin A. Methicillin-resistant Staphylococcus aureus colonization and disease severity in atopic dermatitis: a cross-sectional study from South India. Indian J Dermatol Venereol Leprol. 2014;80(3):229-34. doi: 10.4103/0378-6323.132250. [PubMed: 24823400]. 